\title{
Research on the Application of Information Entropy in Experience Design
}

\author{
Haiyan $\mathrm{Cao}^{1, \mathrm{a}}$ \\ 1 Lanzhou Jiaotong University, P.O. Box 105, Anning Xilu 88, Lanzhou, Gansu, China \\ 34052115@qq.com
}

Keywords: User Experience; Experience Design; Information Entropy; Logical Cognition ; Sentimental Mood

Abstract. By taking experience design as the research center, the paper introduced concept of information entropy into the field of user experience cognition of industrial products, studied the effect of the information entropy on experience design from two aspects of physical cognitive and psychological experience, put forward to limiting factors as leading factors in users' experience. To enhance the information's complexity by increasing the information entropy would be an effective way to strengthen the perceptual experience, which was demonstrated with examples. To research on the application in experience design of information entropy, it could help to explore the user's physiological and psychological perception experience, and fully realize the value of information transmission.

\section{Introduction}

With the changes in the way the economic subject exists, human society through the product economy era, commodity economy era, service economy era, and the rapid to the era of experience economy era. Experience economy era regards meeting the degree of customer experience as the standard, and shapes the consumer process with user self-experience as the center. Therefore, the study of user experience has been elevated to an unprecedented height, and the experience design concept came into being.

The user's experience psychology includes cognitive experience and emotional experience [1]. Albern [2] believes that the user experience psychology covers all aspects of user interaction with the product, including the user experience, the understanding of the product, the degree of completion of the target and the degree of adaptation to the environment in the process of using the product. As a result of a wide range of disciplines, knowledge system is huge, so in-depth study of the user all experiences state and satisfaction in the process of using products or product systems, the need to combine the multi-disciplinary areas and a variety of research methods [3]. Hassenzahl and so that the user experience is also in the user interaction with the product to achieve, is the user's internal state system characteristics and external context of the product of the interaction [4], this experience is not only refers to the user in a particular environment, the behavior of the state and Behavior results, but also contains all of its experience process, its connotation into the psychological and emotional factors for the study [5], that is, people and products, people and the environment, products and the environment how to "contact" and "play a role "

To sum up, the study of user experience is based on the psychological classification of user experience, that is, rational cognition and perceptual cognition. At the same time, in addition to the impact of the product or system, the user awareness includes the situation of using the product. This shows that the interaction of information interaction between multi-elements, "people - machine environment" mutual influence and constraints, the formation of community. It is of great significance to study the relationship between information dissonance (information entropy) and cognitive experience on the premise of specific cognitive purpose and to improve the user's cognitive efficiency and stimulate the user's multi-dimensional perception channel. 


\section{User Experience and Experience design}

Ordinary psychology will be summarized as a way of perception, refers to the human behavior in the practice of psychological behavior, is the subject of the object to stimulate an internal response. User experience is a kind of purely subjective thinking activity and behavior, this psychological feeling has a strong subjectivity, the use of products or services in the process of establishing, that is, individual differences, which leads to the real experience of each user, cannot be fully simulated by other means. However, it is necessary to study the cognitive characteristics of a clear user group and apply the research results of the user group physiology and psychological cognition to the experience design process, so as to achieve the purpose of obtaining a better experience and realize its practical application value.

Experience design is to participate in the design of the consumer, in the design of the service as a "stage", the product as a "props", the environment as a "set" [6] to consumer users a better experience as the goal, And operating habits, in the product or service into more human elements, to meet the practicality and ease of use [7]. To meet the interaction between man and machine characteristics, and fully explore the user in the operation of the perception experience, user experience design needs to solve the key issues.

\section{Information Entropy}

Entropy is a term created by the German physicist Rudolf Clausius in 1850 and refers to the unit of measurement of the sum of the energy that can no longer be converted into work. "Entropy is in the thermodynamic system Used to indicate the degree of chaos of the thermodynamic system is a kind of irregular, is the lack of organizational structure in the situation. In 1948, Claude E.Shannon introduced the concept of entropy into the field of information theory, and defined the information (entropy) as the probability of occurrence of discrete random events. Norbert Wiener, the founder of American cybernetics, argues that "information is what we adapt to the outside world and makes this adaptation the content and name that is exchanged with the outside world in the process that the outside world feels."

The information reflects the nature of the existence of things. The characteristic of things is that it has a certain orderly structure or organizational model, the nature of the structure determines the nature of things. The information theory uses the information entropy concept to measure the degree of order of the system, which indicates the degree of reduction or elimination of the disorder state after the information is obtained. The higher the degree of order of a system, the smaller its entropy, and the greater the amount of information contained.

In the process of information dissemination, information entropy can be defined as the probability of occurrence of particular information [8]. The application of the concept of "information entropy" to the design information system can help to further understand and explain the effective transmission of design information and the value of information experience. From the perspective of information dissemination, the higher the probability of the emergence of a message, the higher the degree of information being quoted, the stronger the communication of the information, and the greater the probability of the user generating the experience value after the communication. Therefore, as a measure of the value of information standards, information entropy can fully reflect the value of information.

\section{Information Entropy and Artistic Experience Perception}

Art activities constituted by three links the artistic creation, artistic works and artistic acceptance. In addition to the artistic works presented in kind, the art of the creation and acceptance process are 
based on their own experience perception ability to the initiative of the subject matter as the deciding factor. The difference is that the artistic creation is done by the artist or the designer, through the experience and thinking of the visual symbol of the reality, through a specific art design language expression; and the latter through the visual perception system to experience and perception of art works, Is a way to interpret the works of art based on the way, is a visual symbol of a self-subjective interpretation of emotional experience and evaluation. These two action processes are centered on artistic experience and are used for the purpose of effective communication and acceptance by art or design information.

Information theory and artistic creation closely linked and combined initially began in the study of Arnheim. Anneheim based on the shape of psychology on the study of perception, that works of art is a perception field [9]. Creators through the arrangement of various shapes, colors, sports and other elements, so that the perception of the interaction, so that the ultimate internal power to achieve a balance. France and Henaut [10] research entropy in the application of product design, in-depth discussion of the relationship between entropy and art, put forward the entropy of the works and the complexity is proportional to the possible emergence of different order composition patterns, the information contained The amount will be more. Petrov [11] used the information entropy method to analyze the creative patterns and style stability of the paintings of the three Soviet Russian artists, and Tsai [12] introduced this method into the study of web design in order to explore the digital museum The weight value of the influence of various characteristic elements on visual perception. This shows that the concept of entropy in information theory has a quantitative influence on the art and design behavior of visual perception as the dominant perception. The information entropy is worth changing and will directly affect the subjective experience of the subject of visual perception. It includes the product main body, the surrounding environment, service content and other experience design elements of cognitive changes.

\section{Application of Information Entropy in Experience Design}

As one of the founders and main representatives of information theory aesthetics, the French scholar Abraham Moles put forward the concept of "aesthetic information" on the basis of information classification of general information theory, divides the information into technical information, semantic information and Aesthetic information three levels. Technical information refers to the material properties of information; semantic information refers to the actual content of information; aesthetic information is a new form of information superimposed on semantic information (Abraham Moles, 1958). The classification of design information is also based on this information classification research theory. Design information is a complex set with openness and mobility characteristics, technical information, semantic information and aesthetic information constitute the three levels of design information.

Information entropy is the determination of environmental factors uncertainty. As the measure of disordered state, the greater the entropy of the system is, the greater the degree of activity and freedom, the smaller the organizational and predictability of the system. Thus, the information can be defined as the number of options when predicting results or the number of new information is proportional to the predicted results. A complex situation with a variety of selectivity and possibilities contains a large degree of uncertainty in the information, so the information entropy is large. In other words, the effective prediction of the results in complex situations requires more facts or context than predicting results in simple contexts. Thus, the information has a function of reflecting the number of selection methods. The more information is unknown in a situation, the greater the entropy of information, the more opportunities for the operation of the situation, which is particularly useful for aesthetic information.

In the cognitive experience to enhance the orderly information, effective communication of semantic information. The acquisition of semantic information is based on the rational cognition in the process of human experience, and obtains the information content through rational thinking such as 
deductive reasoning, logical analysis and data verification. The technology function is the leading product design, so as to realize the correct identification and effective operation for the purpose, to effectively transmit the extension semantic information as a way.

The external form of industrial products to a certain extent can be intuitive to show the essential attributes of the product, it can explain the type and function of the product, or product structure. Then the form of such information is called out of the form of the extension of the semantics. Extensional semantics refers to the use of morphological elements or things to express the purpose of use different product identification, different functions, different specifications, that is, the physical properties of the product [13]. In the process of product operation, the designer can limit the user behavior by setting restrictions or guidance, reduce the user's operating options, the essence is to reduce the product information noise, save operating time and improve operational accuracy. At the same time, the designer will use the user's psychological model to the industrial product design field, which will greatly shorten the user's cognition and operation time, improve the efficiency of human-computer information interaction, meet the rational cognitive needs and realize the practicality and ease of use.

To improve the information entropy is an effective means to enhance the emotional experience design. With the aesthetic information as the leading information of the industrial design, to meet the core function of the creation of the premise, the greater the information entropy, the design of the elements of the higher degree of activation, the greater the impact between each other, the more information users selectivity The deeper the degree of perceptual experience.

Taking competitive characteristics of the dominant industrial products - virtual game products as an example, computer game development and design is based on in-depth study of user perception system, based on the role of people, human-computer interaction, environmental integration and other aspects to fully meet the user's physical and psychological experience. American writer Verlyn Klinkenborg evaluation of the main psychological basis of computer games is to play the game produced by "through a door into another world instinctive feeling." In interactive games, the development of the story depends on both the activities of the game user and the storyline. From the micro-level analysis, the virtual space-time "things" for many discrete rules of the collection, the plot and the story is the player and the rules of the game in the gradual construction of the interaction. The game itself will design a variety of ways for the players to choose their own, lead to different rules to make the user experience different. The rule is characterized by its limitation rather than decisiveness, which is based on the relationship, not the object-based, and the rules form the basic grammar of the digital virtual world. The rules define the behavioral characteristics of the interaction between people and things in an open way, and the user creates the "things" of their own under the guidance of the rules, that is, different choices mean different "story" experiences. The greater the ability of the game user to set the game "story", the greater the uncertainty of the information, the greater the information entropy, the greater the amount of information needed to solve the problem, the more intense the experience of human-computer interaction The The innovation of the rules is also one of the basic ways to innovate the virtual experience design [14]. This is also the game product and film, television, radio and other products exist between the nature of the difference, that is, the event has no control of the ability to choose, the consumer's subjective initiative cannot participate. People are more inclined to choose in the game and the "game story" to re-create, this sense of participation and peak experience in the game to get the ultimate performance. This sense of self-satisfaction and sense of accomplishment is an important way of emotional experience.

In product design, the DIY design is a typical design type that increases the design information entropy. DIY is the English "does it yourself" abbreviation form, literally translated as "DIY". Due to combining the creative thinking performance of consumers, it made the functionalism-based DIY design at the same time a new culture and a "do-it-yourself" new way of life, and formed a huge market space. DIY provides the user's own product of the possibility of the main product, making the user's creativity and subjective initiative have been a great play, the user's right to choose the right to further in-depth development. More importantly, the user in the hands-on operation and the realization of the 
process, the sense of accomplishment sense of psychological and satisfaction to meet the psychological needs of the experience can be achieved. DIY design is in the product design to verify the design information entropy maximization principle of scientific and effective typical design.

\section{Conclusions}

1.One of the objective laws of the development of things is to fight for the greatest freedom in the restraint. Any substance system will be subject to external conditions, the system will always maintain a certain degree of freedom or diversity, this diversity directly lead to the system elements of the rich and free state.

2.In the design field, information entropy is an effective measure of the richness of the design factors. 3.To meet the design of the core functions as the premise, effectively inhibit the confusion of information expression; improve the user to accept the timeliness of semantic information to achieve the correct user operations, or to achieve design reflection.

4. By constructing the rules of design grammar, the disorder and unpredictability of the elements are enhanced, that is, by adding the information entropy to enhance the product user experience, to meet the user's psychological motivation needs.

\section{Acknowledgements}

This work was financially supported by the Gansu Social Science Foundation (YB073).

\section{References}

[1] (In Chinese)Quan Lu, Jing Deng: Intelligence Theory and Practice Vol. 33 (2014), p. 78.

[2] Alben L: Interactions, Vol. 3 (1996), p. 11-15.

[3] Makela A, Fulton Suri J. Proceedings of the International Conference on Affective Human Factors Design (2011).2001, p. 387-394.

[4] Hassenzahl, M.,Tractinsky, N: Behavior and Information Technology Vol. 25 (2015), p. 91-97.

[5] (In Chinese)Xiaoqing Li: Intelligence Theory and Practice Vol. 28 (2016), p. 763-767.

[6] Shedoff Nathan:Experience Design,Indianac New Riders Publishing(2001), in press.

[7] (In Chinese)Zijian Liu: Packaging Engineering Vol. 33 (2015), p. 76-79.

[8] (In Chinese)Li Chen, Haihai Zhou: Journal of graphics Vol. 33 (2016), p. 31-37.

[9] Arheim R:Entropy and Art-an Essay on Disorder and Order. University of California Press(1971).

[10] France M.M: Entropy and stability in painting, Vol. 27(2014), p.219-221.

[11] Petrov V.M: LEONARDO, Vol. 35(2002): p.197-202.

[12] Tsai Tzu-Wei, Chang Tien-Chun, Chuang Ming-Chuen: International Journal of Design, Vol. 2 (2008), p. 55-66.

[13] (In Chinese)Linghao Zhang: Product Semantics,Chinese Building Industry Press(2009), Beijing.

[14] (In Chinese)Lie Zhang: Decoration Vol. 199 (2009), p. 84-85. 